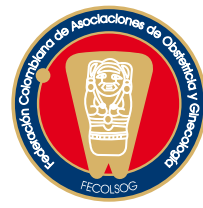

EDITORIAL

\title{
"CONTAR CADA MUERTE, CADA MUERTE CUENTA..." (Iniciativa regional de reducción de la mortalidad materna, OPS 2005)
}

\author{
"Counting each dleath, each death counts..." \\ (PAHO regional initiative for reducing maternal \\ mortality, 2005)
}

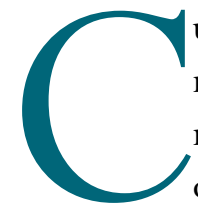

uando en el año 2006 Nacer analizó las muertes por hemorragia obstétrica ocurridas en el departamento de Antioquia durante los dos años anteriores, se insistió en la necesidad de fortalecer tres prácticas que ya habían sido identificadas (en otras latitudes y por otros investigadores) como cruciales para disminuir la mortalidad y morbilidad por esta temible complicación.

En primer lugar, el manejo activo del tercer período del parto, que hasta ahora ha sido la práctica más importante para disminuir el riesgo de hemorragia posparto. ${ }^{1}$ En Antioquia se han logrado avances en ese sentido pero aún no se alcanza un estándar del 100\%. En el año 2007, en un total de 2083 historias clínicas evaluadas se encontró un porcentaje de manejo activo del alumbramiento del $68 \% .^{2}$ No dispongo de cifras objetivas recientes de cuál es la frecuencia de aplicación de esta práctica en el país, pero considero imperativo hacer esta evaluación e incluirla como un estándar de obligatorio cumplimiento. Con la evidencia disponible no se concibe que una mujer sea atendida en una institución de salud en Colombia y no reciba los beneficios de esta práctica.

En segundo lugar, la vigilancia adecuada del puerperio inmediato, entendida como el control de signos vitales y la vigilancia cada 15 minutos del sangrado y la contracción uterina durante las dos primeras horas del posparto, tal como fue definida desde el año 2003 por el Consenso de la Federación Internacional de Matronas y la Federación Internacional de Obstetras y Ginecólogos. " "La falta de atención al cuidado posparto es una tragedia ignorada en los países en desarrollo". Esta frase publicada hace más de 12 años, describe muy bien lo que continúa ocurriendo en el departamento de Antioquia como lo ha hecho evidente la vigilancia epidemiológica, y es probable que en otras regiones del país suceda algo similar. En el año 2007, en 2082 historias clínicas evaluadas en el departamento, el porcentaje de cuidado adecuado en el posparto inmediato fue del 19\%. ${ }^{2}$ Esta cifra habla por sí sola y explica en parte por qué aún en Medellín, con la tecnología más sofisticada a la mano, se siguen presentado muertes por hemorragia posparto.

Y finalmente el "Código Rojo" que presentamos hoy: una propuesta para la atención sistemática y organizada de la mujer con choque hemorrágico de origen obstétrico. Ésta es una guía que tiene la ventaja de ser producto no sólo de la revisión de la literatura, sino del análisis de los factores identificados en el proceso de atención de las mujeres que fallecieron por hemorragia en el departamento en los últimos cuatro años. Éste es el aprendizaje más importante que queremos compartir: "sólo el hecho de ir más allá de los números", ${ }^{5}$ que incluye conocer todas y cada una de las historias del camino hacia la muerte recorrido por estas mujeres, mirar los factores comunes, pero también las diferencias y convertir esta información en acción, permitirá que en cada uno de los rincones de Colombia se avance en el logro de la meta 6 de los Objetivos de Desarrollo del Milenio. Por ello, pre- 
sentamos el Código Rojo sólo como una contribución al abordaje de la morbilidad y mortalidad materna por hemorragia. No es la solución completa, pero estamos convencidos que es una parte sustancial de ella.

Son muchos los retos pendientes en Antioquia y creo que también en el resto del país. El parto no institucional es uno de ellos. Aunque en el departamento, el porcentaje de parto institucional pasó de 97,6\% a 98,6\% entre los años 2004 y 2007, ${ }^{6} 27$ mujeres fallecieron durante, o poco después, de tener un parto en casa, lo que significa un 12,3\% del total de las muertes maternas ocurridas en el mismo período. En el año 2007, 22 muertes maternas ocurrieron después de un parto vaginal, siete de los cuales fueron atendidos por partera tradicional, lo que representa un $33 \%$.

Otras tareas pendientes y urgentes tienen que ver con la necesidad de mantener proveedores calificados y motivados en sitios apartados donde no existen las mínimas condiciones laborales de estímulo. Los esfuerzos deben orientarse hacia el logro de crear o fortalecer entornos habilitantes en los hospitales que atienden a las personas de las comunidades más vulnerables, especialmente cuando aquellos no disponen del personal ni de los recursos para avanzar en los procesos de garantía de la calidad del servicio. Otro reto, es asegurar mecanismos eficientes de referencia y contrarreferencia, en un sistema de salud que se caracteriza por su fragmentación. Por esto, dar vida a la atención primaria renovada, es un desafío urgente, que se visualiza como una respuesta efectiva a la necesidad de fortalecer las capacidades de las mujeres y sus familias y de vincularlas junto a las comunidades a los procesos de planeación y atención en salud.

Sólo con este enfoque renovado de la atención primaria en salud, que incluye poner a disposición de las mujeres, atención de la mejor calidad, accesible y culturalmente aceptable, será posible avanzar hacia la superación de los obstáculos que limitan o impiden el uso de los servicios de salud y hacen que en Colombia, en pleno siglo XXI, muchas mujeres sigan muriendo en sus casas por causas relacionadas con el embarazo y el parto. Asimismo, se lograrán enfrentar eficientemente estos retos, si se cuenta con un sistema de vigilancia epidemiológica activo y fuerte en los niveles locales, pero con capacidad de integración y coordinación entre y con los diferentes niveles del sistema de salud. Un sistema que busque, analice, planee, ejecute, evalúe y retroalimente. Esto sólo será posible si se da en el marco de una política de salud pública de atención primaria donde los principios de la equidad e igualdad sean los que prevalezcan.

A la memoria, de todas las mujeres que murieron en el mundo como consecuencia de complicaciones en su embarazo, mientras yo escribía esta editorial. .60,120,180?.....Una, cada minuto!

\section{Gladis Adriana Vélez Álvarez, M.D.}

Especialista en ginecología y obstetricia

\section{REFERENCIAS}

1. Prendiville WJ, Elbourne D, McDonald S. Conducta activa versus conducta expectante en el alumbramiento (Revisión Cochrane traducida). En: La Biblioteca de Salud Reproductiva, Número 8, 2005. Oxford: Update Software Ltd. Disponible en http://www.rhlibrary.com. (Traducida de The Cochrane Library, Issue 4, 2004. Chichester, UK: John Wiley \& Sons, Ltd.)

2. Uribe BS, Peña PB, Betancur CL, Restrepo OO (Universidad de Antioquia, Facultad de Medicina, Departamento de Ginecología y Obstetricia, Nacer, Salud Sexual y Reproductiva). Maternidad segura con calidad: informe final de auditoría de la calidad en el municipio de Medellín 2007. Medellín: Secretaría de Salud de Medellín; 2008 Feb. Contrato Nro.: 4700025927.

3. International Confederation of Midwives (ICM) and the International Federation of Gynecology of Obstetrics (FIGO). Management of the third stage of labour to prevent postpartum haemorrhage. J Obstet Gynaecol Can 2003;25:952-3.

4. Li XF, Fortney JA, Kotelchuk M, Glover LH. The postpartum period: the key to maternal mortality. Int J Gynaecol Obstet 1996;54:1-10.

5. World Health Organization. Beyond the numbers: reviewing maternal deaths and complications to make pregnancy safer. Geneva: WHO; 2004.

6. Dirección Seccional de Salud de Antioquia. Estadísticas, nacimientos. Medellín: DSSA. Visitado en 2009 Feb 7. Disponible en: http//www.dssa.gov.co/evitables/ nacimientos.htm 